\title{
Study on a New Ecology Insulating Riser
}

\author{
Zhaoming Liu \\ School of Materials Science and Engineering \\ Dalian Jiaotong University \\ Liaoning, China \\ lzm100@163.com
}

\author{
Yangbo Chi \\ School of Materials Science and Engineering \\ Dalian Jiaotong University \\ Liaoning, China \\ chiyb0810@163.com
}

\begin{abstract}
In order to replace the common ecology insulating riser, the dust of foundry is chosen as insulating-refractory material, sorel gel materials as binder and depending on the characteristic of foam beads that shrinking after heated to research on a new ecology insulating riser. The results show that the new ecology insulating riser has three times of feeding efficiency than expanded perlite ecology insulating riser. Recycling the dust of foundry can not only achieve a green casting, but also save the cost of emissions, transport of dust and purchasing ecology insulating riser.
\end{abstract} saving

Keyword-dust, ecology, feeding efficiency, green casting, cost

In foundry production, in order to avoid the defects such as shrinkage cavity in solidification process of liquid metal, setting riser in the casting mold is one of the important measures. However, poor heat preservation performance, large size and low feeding efficiency of common riser, so the research and application of insulating riser gradually get attention. Insulating riser improves the efficiency of riser feeding by controlling the riser heat loss and the extension of the liquid metal solidification time. It can not only ensure the quality of casting and the yield, but also improves the process yield of casting.

Ecology refers to the state of existence of all living things, and the interlocking relationship between them and the environment. In a word, it's recycle. It is not only a kind of protection of natural resources, also can reduce waste, and let Garbage Mountain in the world a bit less.

\section{THE DEVELOPMENT OF INSULATING RISER IN CHINA}

Researching insulating riser in our country has a history of more than 40 years, now it has more than 10 kinds of ecology insulating riser products, which mainly include composite fiber, floating beads and expanded perlite insulation riser. Composite fiber type thermal insulating riser cost is high and difficult mixing, the ancillary equipment needed for production. Floating beads type thermal insulation riser heat-resisting performance is poorer, and applied in large steel, the corrosion phenomenon happens. Expanded perlite ecology insulating riser strength is low, big breakage and lower fire resistance. The current insulation riser has its own defects, which affect their application scope.

In this paper, the following materials have been chosen with the central idea of green environmental protection and low cost:

(1) Foundry dust. Mainly comes from solid materials mechanical crushing and grinding and burning material, generally comes from the clean workshop, molding workshop and smelting workshop. For factory, foundry dust is a kind of industrial waste polluting the environment, because of its high temperature resistance and low thermal conductivity, it is a production of ecology insulating riser of suitable materials. The refractory temperature of dust used in this paper is greater than $1400^{\circ} \mathrm{C}$ 。

(2) Sorel cement. In 1867, the French sorrel invented this kind of material, it has fast hardening, high strength, fire prevention, wear-resisting, and other advantages. As an inorganic material, it doesn't gives off poisonous gas and excitant odour in the process of casting, so it is a kind of environmentally friendly materials.

(3) Polystyrene foam beads (EPS). It is widely applied and has low price. This material shrinks at $150^{\circ} \mathrm{C}$ or so, which is an important feature used in this paper.

(4)High temperature coating. It is made of silica sol and zircon sand blend according to certain proportion and the coating layer can withstand temperatures up to 1500 $1600{ }^{\circ} \mathrm{C}$.

\section{EXPERIMENTAL MATERIALS AND METHODS}

\section{A. Experimental Materials}

The dust in this experiment is chosen from iron foundry cleaning workshop that containing coating composition, and the coating containing graphite. It means a lot for ecology insulating riser (Formula 1).

$$
\begin{gathered}
\mathrm{C}(\mathrm{s})+\mathrm{O}_{2}(\mathrm{~g})=\mathrm{CO}_{2}(\mathrm{~g}) \triangle \mathrm{H}=-393.5 \mathrm{KJ} / \mathrm{mol} \\
4 \mathrm{Fe}(\mathrm{s})+3 \mathrm{O}_{2}(\mathrm{~g})=2 \mathrm{Fe}_{2} \mathrm{O}_{3}(\mathrm{~s}) \triangle \mathrm{H}=-1648.42 \mathrm{KJ} / \mathrm{mol}
\end{gathered}
$$

Drawn

$$
\begin{gathered}
3 \mathrm{C}(\mathrm{s})+2 \mathrm{Fe}_{2} \mathrm{O}_{3}(\mathrm{~s})=4 \mathrm{Fe}(\mathrm{s})+3 \mathrm{CO}_{2}(\mathrm{~g}) \\
\triangle \mathrm{H}=-467.92 \mathrm{KJ} / \mathrm{mol}
\end{gathered}
$$

By above formula, you can see that the reaction of graphite and iron oxide can release a lot of heat, making the ecology insulating riser have the effect of heat insulation riser, which further improve the performance for keep temperature. The main chemical components of dust are shown in table 1. 
TABLE I. TABLE 1 CHEMICAL COMPOSITION OF DUST

\begin{tabular}{ccccccccc}
\hline $\begin{array}{c}\text { Chemical } \\
\text { composition }\end{array}$ & $\mathrm{SiO}_{2}$ & $\mathrm{Fe}_{2} \mathrm{O}_{3}$ & $\mathrm{C}$ & $\mathrm{Al}_{2} \mathrm{O}_{3}$ & $\mathrm{CaO}$ & $\mathrm{MgO}$ & $\mathrm{K}_{2} \mathrm{O}$ & $\mathrm{Na}_{2} \mathrm{O}$ \\
\hline Mass fraction /\% & 50.3 & 18.6 & 13.1 & 4.3 & 4.6 & 2.5 & 2.7 & 3.4 \\
\hline
\end{tabular}

\section{B. Experimental Methods}

\section{1) Experimental principle}

The heat preservation principle of ecology insulating riser: according to heat transfer theory, there are three kinds of heat transfer that radiation, conduction and convection. While for riser, especially blind riser, conduction is the mainly transitive manner. Thermal conduction within the material is the embodiment of the collision of molecules, by high energy level to low energy transfer. As a result, the effect of heat transfer of amorphous material is lower than that of crystal materials. Big porosity or small bulk material has good heat preservation effect.

In this paper, the new ecology insulating riser, according to the principle mentioned above, is mixed with foamed beads that shrink after heated, then form a closed hollow in riser wall (Fig. 1). The hollow can increase the porosity of riser, at the same time the independent closed cavity can prevent the formation of convection and further improve the insulation performance of riser. This method has obvious advantages over foam. The cavities formed by foam are of various sizes and nor evenly distributed. If the improper control of time, foaming agent can evaporate that lead to the small number of cavity and polluted the work environment at the same time. In the method designed in this paper, the size of cavity can be adjusted by choosing foam beads of different size.

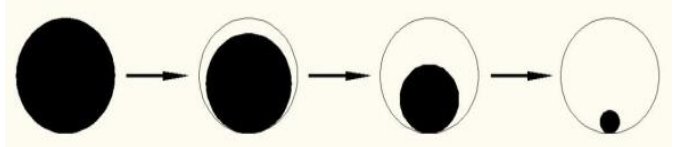

Figure 1. Foam beads shrinking process

2) The method of making ecology insulating riser

Specific production process is the proportion of 30 brine, ling magnesia, industrial dust, polystyrene foam according to 1:1.3:0.3:0.1 then inject the paste into the mould and vibrantly compact. After 3 days' maintenance, coat the riser with a high temperature coating layer of $1 \mathrm{~mm}$ thick. Finally, dry it for 5 hours.

\section{THE EXPERIMENTAL RESULTS AND ANALYSIS}

\section{A. The Analysis of the Heat Preservation Performance of Ecology Insulating Riser}

In figure 2 you can see, after pouring, in the riser wall formed a large and uniform hollow and each hole closed and independent with each other. Testing ideas realized. Upper at the same time, you can also see a lot of not melt shrinkage bubble beads from the picture about a quarter of the thick and, further confirmed that the new ecology insulating riser has good heat preservation performance.

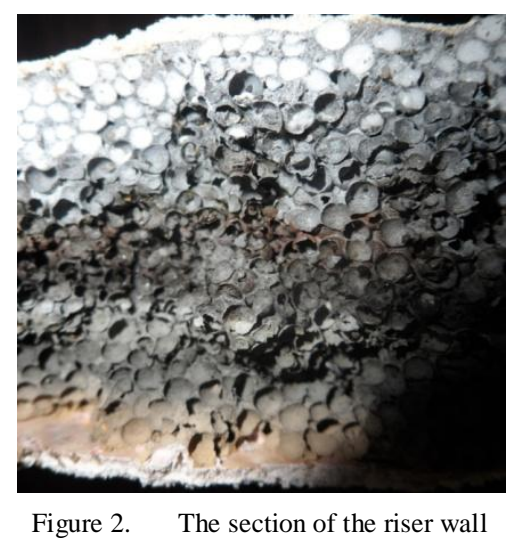

\section{B. The Analysis of the Efficiency of Ecology Insulating Riser Feeding}

In this paper, the experiment was conducted on the foundry production line, production of the parts are cover. Take the insulating riser used in factory as a reference, on the two kinds of insulating riser feeding efficiency comparison. Compare the feeding efficiency of the two kinds of insulating risers. The test result is shown in figure 3.

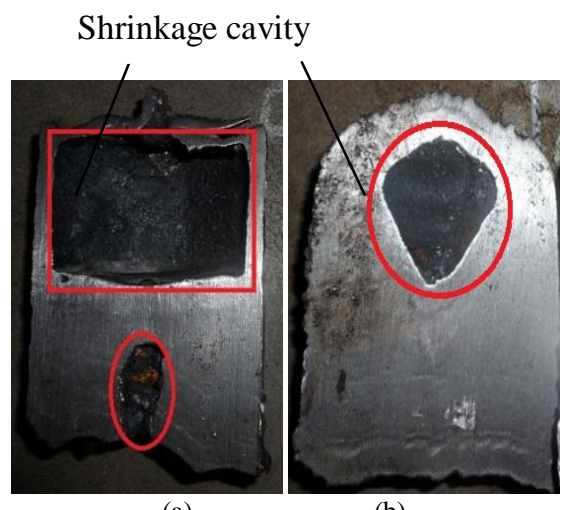

(a)

(b)

Figure 3. The section of the riser

The new ecology insulating riser and the reference insulating riser have exactly the same size. You can see from the figure 3, the new ecology insulating riser (a) formed the ideal flat cylindrical thin shell shrinkage cavity, and the reference insulating riser (b) did unideal taper shrinkage cavity. At the same time as you can see the shrinkage cavity volume of the new ecology insulating riser is very big, the equivalent of three times as many as reference insulating riser. The reason of the formation of new shrinkage cavity is that after the riser formed the initial chilled layer, the good heat preservation effect of the riser, so chilled layer don't thicken. With falling liquid level, finally casting no longer need feeding and liquid level stops falling.

We also observed that the new ecology insulating riser root has shrinkage cavity area, but no defective parts. Through the analysis, the reason is that the new ecology insulating riser size is too large that forms a thermal center in the root. While meeting the needs of the feeding of 
casting, there is still a lot of liquid metal in the region. Finally, the shrinkage cavity formed in the region. So, according to the property of the new ecology insulating riser, the smaller size can reach the effect of the insulating riser used by factory, so as to improve the process yield of casting.

Through calculation, the new ecology insulating riser has three times of feeding efficiency than expanded perlite insulating riser.

\section{Analysis of the Surface Morphology of Ecology Insulating Riser}

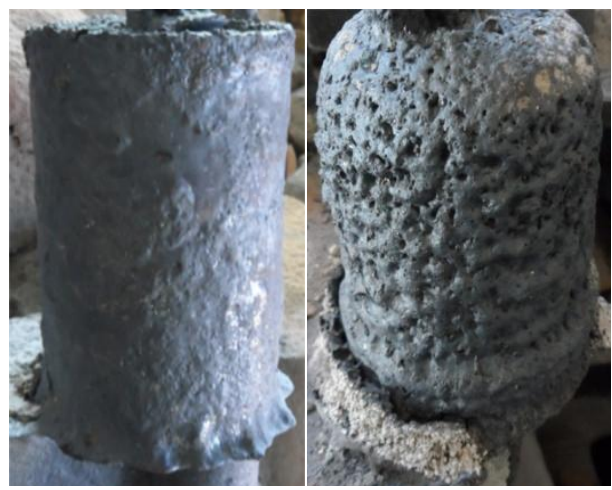

(a)

(b)

Figure 4. The surface topography of riser

We can see from figure 4, the ecology insulating riser (a) has smooth surface and neat appearance, and the surface of insulating riser (b) used by factory is roughness. Under the same casting conditions, this difference reflects the different solidification of molten metal. Riser of rough surface, the molten metal solidification method is volume solidification. In this way of solidification, feeding channel will be blocked that leads to not reach the casting needs. Riser of smooth surface, the molten metal solidification method is layer by layer solidification that can ensure the unimpeded feeding channel.

Observing the root of riser you can see that the new ecology insulating riser is very clean, no debris, while insulating riser of the factory forms ceramic material. This kind of material has high temperature resistant, stable chemical properties and high hardness. It is difficult to remove, and significantly influence the process of cutting riser.

\section{CONCLUSION}

Through practical production we can get the following conclusion:

(1) Using the foundry dust as insulating-refractory material can significantly improve the insulated performance of riser. Do local resources, waste recycling achieve green casting.

(2) The experimental that mixing ecology insulating riser with foam beads to reinforce the insulated performance of riser is entirely feasible.

(3) The new ecology insulating riser is easy to be clean and facilitates the subsequent cleanup work.

(4) In the case of guarantee the feeding needs of castings, decreasing the size of new ecology insulating riser can improve the process yield of castings.

\section{REFERENCES}

[1] Chen Rushu, Miao Miao. The Influence of Composite Blister on the Properties of Porous Insulating Riser [J]. Journal of Dalian Jiaotong University,2009,30(3):48 51.

[2] Kou Fenghe, Zhai Guanhua. The Application of Ecology Insulating Riser [J]. Foundry, 1993, 12(2):2 3.

[3] Zhao Yuhua, Hai Jie. A Study on Insulating Riser and Its Application in Cast Steel Items [J]. Journal of Shenyang Institute of Aeronautcal Engineering, 2000, 17(1):34 36.

[4] Xiao Bin, Qiu Zhanhong. Experimental Study of Proportion of Magnesium Oxchloride Cement [J]. Journal of Taizhou University, 2012, 34(3):45 50.

[5] Wang Zuoli. The Application of Heat Preserving Casting Head in Foundry Production [J]. Research Studies on Foundry Equipment, 2011, (4):39 40

[6] Chou Xingrong, Lei Ping. Test and Performance Analysis of Common Insulating Riser [J]. Foundry, 2010, 59 (5):513 515. 